\title{
Resonant sideband extraction: a new configuration for interferometric gravitational wave detectors
}

\author{
J. Mizuno, K.A. Strain, P.G. Nelson, J.M. Chen, R. Schilling, A. Rüdiger, \\ W. Winkler and K. Danzmann \\ Max-Planck-Institut für Quantenoptik, Ludwig-Prandtl-Strasse 10, W-8046 Garching near Munich, Germany
} Received 10 December 1992; revised manuscript received 27 January 1993; accepted for publication 15 February 1993
Communicated by J.P. Vigier

\begin{abstract}
We introduce a new Fabry-Perot based interferometric gravitational wave detector that, compared with previous designs, greatly decreases the amount of power that must be transmitted through optical substrates to obtain a given light power in its arms. This significantly reduces the effects of wavefront distortions caused by heating due to absorption in the optics, and allows an improved broadband sensitivity to be achieved.
\end{abstract}

To obtain a good sensitivity in long-baseline interferometric gravitational wave detectors, one requires high light power in the arms of the interferometer to increase the photon-statistic limited signal-to-noise ratio. In the standard Fabry-Perot configuration, which consists of a Michelson interferometer having a Fabry-Perot cavity in each arm [1], this can be done by increasing the finesse of the cavities (made possible by the availability of very low-loss mirror coatings ). The storage-time for the signal sidebands must, however, be kept short enough to give the desired detection bandwidth (since cavities act like low-pass filters). This determines what is referred to as the storage-time limit. A high laser power, or a "power recycling mirror" [1], must then be used to compensate for the limitation that the storage-time puts on the power enhancement in the arm cavities. To obtain a sensitivity and a bandwidth which are desirable in future advanced detectors, one is then required to have extremely high light power incident on the beamsplitter, potentially in excess of $10 \mathrm{~kW}$. Thermally induced lensing in the beamsplitter and mirror substrates will make it exceedingly difficult to reach this power $[2,3]$.

By using the principles of coupled cavities, it is possible to increase the finesse of the arm cavities beyond

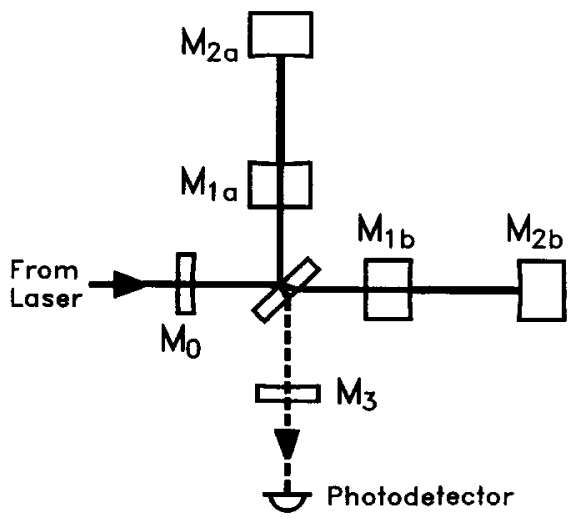

Fig. 1. Schematic diagram of the optical configuration of reso nant sideband extraction. The storage-time for the carrier light in the arms is longer than the storage-time limit, whereas the storage-time for differentially generated sidebands is reduced by the existence of the signal extraction mirror $\left(\mathbf{M}_{3}\right)$. 
that given by the storage-time limit, while keeping the storage-time for the signal sidebands consistent with the desired detection bandwidth. This is accomplished by adding a signal extraction mirror $\left(\mathrm{M}_{3}\right.$ in fig. 1$)$ to the standard configuration, the purpose of which is to decrease the storage-time for the signal sidebands. This is not to be confused with a "signal recycling mirror" [4], which was proposed as a means of increasing the signal sideband storage-time. The result is that the new system can obtain very high power in its arms with only very modest power being present at the beamsplitter, thus greatly reducing the heating problem. In principle, the power enhancement in the arm cavities could be so great that no power recycling would be required.

A suitably polarized gravitational wave will phase modulate the light in the arms, and will thus produce upper and lower sidebands on the laser light with a frequency spacing from the carrier equal to the gravitational wave frequency. These sidebands are generated with opposite phase in the two arms. In a perfect interferometer operated on a "dark fringe", the beamsplitter separates these sidebands from any carrier light leaving the arms. The carrier light is directed towards the laser (where it may be recycled by a power recycling mirror $\mathbf{M}_{0}$ ), and the sideband light towards the signal extraction mirror $\mathbf{M}_{3}$. The mirror $\mathbf{M}_{3}$ thus forms a coupled cavity with the cavities in the arms of the interferometer. It can be used to make the apparent reflectivity at the inboard mirrors $\left(M_{1 a}\right.$ and $\left.M_{1 b}\right)$ lower for the signal sidebands than it is for the carrier light. We will refer to the cavity formed by the inboard mirrors and the signal extraction mirror as the signal extraction cavity (SEC).

The frequency response of the interferometer is determined by the properties of the SEC (assuming the length of the arms are fixed by practical considerations). The reflectivity of the SEC on resonance is determined by the reflectivities of the signal extraction mirror and the inboard mirrors, and sets the approximate detection bandwidth and the sensitivity at zero hertz. Both the length (order) of the SEC and its tuning relative to the carrier light affect the shape of response. We classify the responses into symmetric and asymmetric cases. In the symmetric case, the SEC is chosen to be resonant at the carrier frequency, and the upper and lower signal sidebands generated in the arms see the same reflectivity of the SEC. In the asymmetric case, the SEC is slightly detuned from the carrier frequency, and the sidebands see different reflectivities.

First consider the symmetric case. If the length of the SEC is infinitesimal, its bandwidth is broad, and all signal sidebands see the same reflectivity and experience the same phase shift upon reflection from it. Thus, the frequency response of the interferometer has the same shape as in the standard configuration. The reflectivity of the SEC determines the storage-time for the sidebands in the system, and thus the detection bandwidth. The peak sensitivity is also affected, and increases as the square root of this storage-time. As one increases the length of the SEC (by half wavelength steps, to keep it tuned to the carrier frequency), its bandwidth narrows,
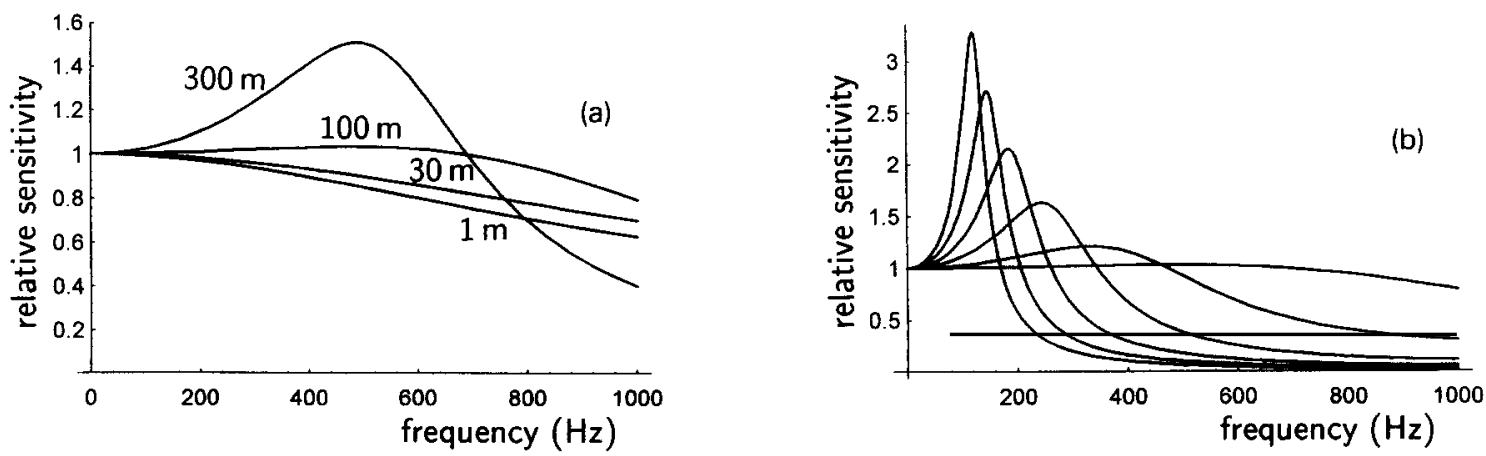

Fig. 2. Typical frequency response curves for the proposed system. (a) Dependence of the frequency response on the length of the SEC in the symmetric case. The curves correspond to lengths of $1,30,100$, and $300 \mathrm{~m}$, respectively. (b) Dependence on the tuning of the SEC to carrier. The broadest response corresponds to the symmetric case, and the others are detuned from it (the asymmetric case). The length of the SEC is $100 \mathrm{~m}$, and the detuning is by steps of $2 \pi / 1000$ in $\delta$. In both figures, the fractional power loss in the SEC is assumed to be $5 \times 10^{-3}$ (dominated by imperfect interference of the beams from the two arms), $t_{1}^{2}=5 \times 10^{-4}$, and $t_{3}^{2}=5 \times 10^{-3}$. The vertical units are arbitrary. 
and the phase of the sideband light reflected from it changes. If we choose the SEC to be undercoupled ${ }^{\# 1}$, the frequency dependence of this phase tends to cancel that associated with the transit time in the arms (over a limited frequency range). This has the effect of flattening the response curve near its peak (at zero hertz), at the expense of making the high-frequency cutoff sharper than in the standard configuration (fig. $2 \mathrm{a}$ ). The bandwidth of the whole system (measured at $-3 \mathrm{~dB}$ of the peak) increases as the SEC is elongated, but typically by a factor less than two. If the length of the SEC is increased further, then a peaked response is obtained. In most cases, however, this is not practical, and narrow-banding is more readily accomplished in the asymmetric case described below. By an appropriate choice of the length and reflectivity of the SEC, a suitable compromise between the sensitivity, bandwidth and flatness of response can be found. Making the bandwidth of the SEC comparable with the desired detection bandwidth provides the flattest response.

In the asymmetric case, one starts with a suitable symmetric response, and tunes the resonance of the SEC slightly away from the carrier frequency (by moving the signal extraction mirror a small fraction of a wavelength). This produces a peak in the response, as illustrated in fig. $2 \mathrm{~b}$. As the detuning continues, the peak moves to lower frequencies, narrows in bandwidth, and increases in height. The bandwidth is roughly proportional to the peak frequency, and the height approximately inversely proportional (for all but the narrowest bandwidths). This dependence on the position of the signal extraction mirror differs from the case of signal recycling, where the peak sensitivity and bandwidth are independent of the mirror's position, and only the frequency of the peak response changes. If both configurations are optimized for the same peak frequency and bandwidth, however, similar frequency responses can be obtained.

To evaluate the frequency response of the system, it is helpful to apply the formalism of Meers [5]. He derives the frequency response for interferometers by considering the cavity in which the signal sidebands are stored. In an interferometer such as ours, this is a three-mirror coupled cavity system, consisting of an end mirror, and a compound output mirror. In this case the end mirror corresponds to $\mathbf{M}_{2 \mathrm{a}}$ or $\mathbf{M}_{\mathbf{2 b}}$, and the compound mirror to the SEC. As a major difference from those considered in ref. [5], our configuration exploits the behavior of the system when the compound output mirror (one SEC) is nearly resonant with the sideband light (ref. [5] treats only the non-resonant case). The following equation gives the ratio of the signal sideband amplitude appearing at the output of the interferometer to the input carrier amplitude incident on the beamsplitter,

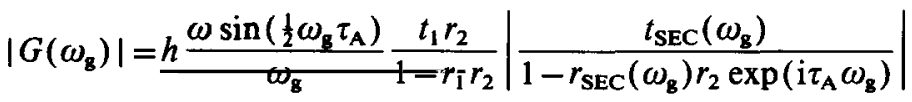

$$
\begin{aligned}
& =h \frac{\omega \sin \left(\frac{1}{2} \omega_{\mathrm{g}} \tau_{\mathrm{A}}\right)}{\omega_{\mathrm{g}}} \frac{t_{1} r_{2}}{1-r_{1} r_{2}} \frac{t_{1} t_{3}}{\left|1-r_{1} r_{2} \exp \left(\mathrm{i} \tau_{\mathrm{A}} \omega_{\mathrm{g}}\right)-r_{1} r_{3} \exp \left[\mathrm{i}\left(\delta+r_{\mathrm{S}} \omega_{\mathrm{g}}\right)\right]+r_{2} r_{3} \exp \left\{\mathrm{i}\left[\delta+\left(\tau_{\mathrm{A}}+r_{\mathrm{S}}\right) \omega_{\mathrm{g}}\right]\right\}\right|} .
\end{aligned}
$$

Here, $h$ is the strain amplitude induced by the gravitational wave. The complex amplitude transmittance and reflectivity of the SEC are $t_{\mathrm{SEC}}\left(\omega_{\mathrm{g}}\right)$ and $r_{\mathrm{SEC}}\left(\omega_{\mathrm{g}}\right)$. The amplitude transmission and reflection coefficients for the $n$th mirror (where "a" and " $\mathrm{b}$ " mirrors are assumed identical) are $t_{n}$ and $r_{n}$. The sum of their squares can be less than one, depending on the model for losses in the system. The angular frequency of the light and the gravitational wave are given by $\omega$ and $\omega_{\mathrm{g}}$, respectively. The round-trip transit time for light in the arms is $\tau_{\mathrm{A}}$ and for the SEC is $\tau_{\mathrm{s}}$. The phase offset of the SEC is given by $\delta$ (equal to zero in the symmetric case). Figure 2 shows the frequency responses obtained by summing over the upper and lower sidebands, taking their relative phase into account. Responses other than the ones described here are possible. Direct comparison of these curves with those for the standard configuration is highly model dependent, so we do not do so here [6]. Optimization will require a detailed analysis which includes the non-ideal aspects of the interferometer.

To choose the mirror reflectivities and separations, one must consider the losses in the system. The important

\#1 In an undercoupled cavity, the amplitude of the light directly reflected from the input mirror is larger than that leaking out from inside the cavity. 
losses are those in the arms, and those in the SEC. The loss in the arms is due to scattering and absorption in the mirror coatings, and it dominates the power loss in the system. It does not directly affect the frequency response. The loss in the SEC is mainly due to imperfect interference of the beams from the two arms, Rayleigh scattering in the optics, and losses at anti-reflection coatings. It limits the efficiency of signal extraction that can be achieved and alters the shape of the frequency response. To optimize the frequency response and sensitivity, the losses in the SEC should be small compared with the transmission of the signal extraction mirror. To achieve this, one may need to increase the transmission of the inboard mirrors $\mathrm{M}_{1 \mathrm{a}}$ and $\mathrm{M}_{1 \mathrm{~b}}$. It should be noted that the resulting reduction in power buildup in the arms can be compensated by a relatively modest power recycling factor (perhaps on the order of ten).

In any proposed configuration optimized for a chosen bandwidth, there is a minimum energy (number of photons) that must be stored in the arms to achieve a given sensitivity. Future detectors aiming for a strain sensitivity of $10^{-22}$ in a $1 \mathrm{kHz}$ bandwidth will need at least $20 \mathrm{~J}$ of $1 \mu \mathrm{m}$ wavelength photons to be stored. With arm lengths of $3 \mathrm{~km}$, this requires an optical power in each arm of $500 \mathrm{~kW}$ \#2. For the standard configuration, the storage-time limit requires the effective number of beams in each arm to be less than 50 , and thus the power incident on the beamsplitter must be at least $40 \mathrm{~kW}$. At such power levels, severe thermal distortions can be expected given the currently available substrate materials [2,3]. In the proposed system, numerical models [6] show that finesse can be increased to approximately 10000 , which would require a power incident on the beamsplitter of only $150 \mathrm{~W}$. Assuming $50 \mathrm{ppm}$ loss per round-trip, the reflectivity of the arm cavities should be approximately 0.65 , and these powers should be obtainable with only a modest power recycling factor. Of course, the power in the arms remains high, and careful consideration must be paid to the thermal effects due to absorption at the mirror coatings. This, however, is common to all proposed configurations that use FabryPerot cavities in the arms.

In conclusion, using this technique of resonant sideband extraction allows Fabry-Perot based interferometers to have long storage-time, very high finesse arm cavities without sacrificing the detector's bandwidth. This makes it possible to have high light power in the arms without requiring high power to be transmitted through any optics. This effectively eliminates one of the dominant distortion problems in the standard configuration, and thus the broadband sensitivity of this configuration should exceed those of previously proposed designs.

The authors would like to thank the gravitational wave group at the University of Glasgow, especially Jim Hough, Euan Morrison, David Robertson, and Harry Ward who all provided helpful insights into the understanding of this system. This work has been supported by the Bundesministerium für Forschung und Technologie (BMFT). One of the authors (JM) would like to thank the JSPS for its support.

\#2 The power actually incident on the mirrors may be less than this if folded Fabry-Perot cavities or delay lines are used.

\section{References}

[1 ] R.W.P. Drever et al., in: Quantum optics, experimental gravitation, and measurement theory, eds. P. Meystre and M.O. Scully (Plenum, New York, 1983) pp. 503-514.

[2] W. Winkler et al., Phys. Rev. A 44 (1991) 7022.

[3] K.A. Strain et al., in preparation.

[4] B.J. Meers, Phys. Rev. D 38 (1988) 2317.

[5] B.J. Meers, Phys. Lett. A 142 (1989) 465.

[6] J. Mizuno et al., to be published. 